\title{
CONVOLUTION THEOREMS OF TITCHMARSH TYPE ON DISCRETE $\mathbf{R}^{n}$
}

\author{
by YNGVE DOMAR
}

(Received 21st March 1988)

\section{Summary}

This paper contains results related to Titchmarsh's convolution theorem and valid for $\mathbf{R}_{d}^{n}$, the additive group of $\mathbf{R}^{n}$ with the discrete topology. The method of proof consists in transferring the problem to $\mathbf{R}^{n}$ with the usual topology by a procedure which has been used earlier, for instance in Helson [3].

In Section 1, the classical support theorems are generalized to $\mathbf{R}_{d^{*}}^{n}$ In [1], Titchmarsh's convolution theorem [6] on $\mathbf{R}$ was generalized to convolutions of functions belonging to certain weighted $\mathbf{L}^{p}$-spaces on $\mathbf{R}$. Section 2 contains a corresponding generalization to weighted $l^{2}\left(\mathbf{R}_{d}\right)$.

It should be observed that convolutions of elements $f$ and $g$ in $l^{1}\left(\mathbf{R}_{d}^{n}\right)$ can be interpreted as convolutions of bounded discrete measures on $\mathbf{R}^{n}$. Hence, in that case the support theorem (Theorem 4.33 of Hörmander [5]) is directly applicable to give the results of our Theorems 1 and 3. So the novelty in our theorems lies in the fact that they apply for instance to the case when it is only assumed $f, g \in l^{2}\left(\mathbf{R}_{d}^{n}\right)$, together with support conditions. It is not known whether it suffices to assume $f \in l^{1}\left(\mathbf{R}_{d}^{n}\right), g \in l^{p}\left(\mathbf{R}_{d}^{n}\right)$, when $p>2$.

\section{Theorems on the convex hull of the support}

The points on the vector space $\mathbf{R}^{n}$ are denoted $\lambda=\left(\lambda_{1}, \ldots, \lambda_{n}\right)$. On $\mathbf{R}^{n}$ we will alternate between the usual and the discrete topology. In the latter case the space is denoted $\mathbf{R}_{d}^{n}$. The space $\mathbf{R}_{d}^{n}$ is a discrete group under addition, and its dual group is $b \mathbf{R}^{n}$, the Bohr compactification of $\mathbf{R}^{n}$. For $t \in \mathbf{R}^{n}, e_{t}$ denotes the element of $b \mathbf{R}^{n}$, which corresponds to the character $\lambda \mapsto \exp (i \lambda t)$ on $\mathbf{R}_{d}^{n}$. The set of elements $e_{t}$ forms a Borel measurable subgroup $\mathbf{R}_{o}^{n}$ of $b \mathbf{R}^{n}$, since $t \mapsto e_{t}$ is continuous. The Fourier transformation $\mathscr{F}$ from $\mathbf{R}_{d}^{n}$ to $b \mathbf{R}^{n}$ is formally defined by

$$
\hat{f}(x)=\mathscr{F} f(x)=\sum_{\lambda \in \mathbf{R}_{a}^{n}} f(\lambda)\langle x, \lambda\rangle, x \in b \mathbf{R}^{n},
$$

with the inverse 


$$
f(\lambda)=\mathscr{F}^{-1} \hat{f}(\lambda)=\int_{b \mathbf{R}^{n}} \hat{f}(x)\langle x,-\lambda\rangle d m(x), \lambda \in \mathbf{R}_{d}^{n}
$$

where $m$ is the normalized Haar measure on the compact group $b \mathbf{R}^{n}$. For convenience we assume in the sequel that all functions in $L^{1}\left(b \mathbf{R}^{n}\right)$ are chosen Borel measurable.

Let $\hat{f} \in L^{1}\left(b \mathbf{R}^{n}\right)$. Then $(x, t) \mapsto \hat{f}\left(x+e_{t}\right)$ is a Borel function on $b \mathbf{R}^{n} \times \mathbf{R}^{n}$. We put

$$
\hat{f}\left(x+e_{t}\right)=\hat{f}_{x}(t),
$$

and observe that $\hat{f}_{x}$ is Borel measurable on $\mathbf{R}^{n}$ for every $x \in b \mathbf{R}^{n}$. Taking into account the invariance of $m$, Fubini's theorem gives

$$
\int_{b \mathbb{R}^{n}} \int_{\mathbf{R}^{n}}\left|\hat{f}_{x}(t)\right|(1+|t|)^{-n-1} d t d m(x)=\int_{b \mathbb{R}^{n}}|\hat{f}(x)| d m(x) \int_{\mathbb{R}^{n}}(1+|t|)^{-n-1} d t<\infty
$$

where $d t$ stands for integration with respect to the $n$-dimensional Lebesgue measure. Hence there is a $\mathbf{R}_{0}^{n}$-invariant Borel measurable set $E$ with $m E=1$ such that

$$
\int_{\mathbf{R}^{n}}\left|\hat{f}_{x}(t)\right|(1+|t|)^{-n-1} d t<\infty
$$

whenever $x \in E$. Thus, if $x \in E$, then $\hat{f}_{x} \in \mathscr{S}^{\prime}\left(\mathbf{R}^{n}\right)$, the Schwartz space of tempered distributions, and $\hat{f}_{x}$ has an inverse Fourier transform $f_{x} \in \mathscr{S}^{\prime}\left(\mathbf{R}^{n}\right)$. In this paper it is convenient to use the relation

$$
\phi(\lambda)=\int_{\mathbf{R}^{n}} \hat{\phi}(t) e^{-i \lambda t} d t, \lambda \in \mathbf{R}^{n}
$$

as the formal definition of inverse Fourier transformation on $\mathbf{R}^{n}$.

In the following, for any subset $F$ of $\mathbf{R}^{n}$ or $\mathbf{R}_{d}^{n}$, $\operatorname{ch} F$ denotes its convex hull with respect to the basic vector space $\mathbf{R}^{n}$ and $\bar{F}$ denotes the closure of $F$ in the topology of $\mathbf{R}^{n}$. For any complex-valued $f$ on $\mathbf{R}_{d}^{n}$,

$$
\operatorname{supp} f=\left\{\lambda \in \mathbf{R}_{d}^{n}, f(\lambda) \neq 0\right\},
$$

while the support for functions or distributions on $\mathbf{R}^{n}$ is defined in the sense of distributions.

Lemma 1. Let $\hat{f} \in L^{1}\left(b \mathbf{R}^{n}\right), f=\mathscr{F}^{-1} \hat{f}$. Then

$$
\operatorname{supp} f_{x}=\overline{\operatorname{supp} f} \text {, }
$$

for almost every $x \in b \mathbf{R}^{n}$.

Proof. For any $\hat{\phi} \in \mathscr{P}\left(\mathbf{R}^{n}\right)$, the function 


$$
\hat{g}(x)=\int_{\mathbf{R}^{n}} \hat{\phi}(t) \hat{f}\left(x-e_{t}\right) d t
$$

is defined on $E$, the set where (2) holds. Fubini's theorem shows that $\hat{g} \in L^{1}\left(b \mathbf{R}^{n}\right)$ and

with $\phi$ defined by (3). For $x \in E$, (4) gives

$$
g=\mathscr{F}^{-1} \hat{g}=\phi f,
$$

$$
\hat{g}_{x}(s)=\int_{\mathbf{R}^{n}} \hat{\phi}(t) \hat{f}_{x}(s-t) d t, s \in \mathbf{R}^{n}
$$

and hence $\hat{g}_{x}$ is continuous, and its distributional inverse Fourier transform $g_{x}$ satisfies

$$
g=\phi f_{x}
$$

It follows from (1), applied to $\hat{g}$, that $\hat{g}_{x}=0$ for almost every $x \in E$ if and only if $\hat{g}=0$ almost everywhere on $b \mathbf{R}^{n}$. Hence (5) and (6) show that

$$
\phi f_{x}=0 \text { for almost every } x \in E \text { if and only if } \phi f=0 \text {. }
$$

Let us define $\psi(x)$ as 0 , if $g_{x}=0$, and as 1 elsewhere on $b \mathbf{R}^{n}$. Then $\psi \in L^{1}\left(b \mathbf{R}^{n}\right)$, and is constant on the cosets of $\mathbf{R}_{0}^{n}$. By a known device (see for instance the proof of Theorem 9 in Helson [3]) this implies that $\psi$ is constant almost everywhere on $b R^{n}$. Hence the set where $g_{x}$ vanishes has either measure 0 or 1 , and we can conclude from (6) and (7) that

$$
\phi f_{x} \neq 0 \text { for almost every } x \in E \text { if (and only if) } \phi f \neq 0 \text {. }
$$

The lemma follows easily from (7) and (8) by varying $\phi$ in a suitable denumerable subset of $\mathscr{D}\left(\mathbf{R}^{n}\right)$.

Definition 1. If $f=\mathscr{F}^{-1} \hat{f}, g=\mathscr{F}^{-1} \hat{g}$, with $\hat{f}, \hat{g}, \hat{f} \hat{g} \in L^{1}\left(b \mathbf{R}^{n}\right)$, we define convolution $f * g$ of $f$ and $g$ by

$$
f * g=\mathscr{F}^{-1}(\hat{f} \hat{g})
$$

Theorem 1. Let $f=\mathscr{F}^{-1} \hat{f}, g=\mathscr{F}^{-1} \hat{g}$, with $\hat{f}, \hat{g}, \hat{f} \hat{g} \in L^{1}\left(b \mathbf{R}^{n}\right)$, and with supp $f$ and suppg bounded. Then

$$
\overline{\operatorname{ch} \operatorname{supp} f * g}=\overline{\operatorname{ch} \operatorname{supp} f}+\overline{\operatorname{ch} \operatorname{supp} g} \text {. }
$$

Proof. By Lemma 1 we have, for almost every $x \in b \mathbf{R}^{n}$,

$$
\begin{aligned}
& \hat{f}_{x} \in \mathscr{S}^{\prime}\left(\mathbf{R}^{n}\right), \operatorname{supp} f_{x}=\overline{\operatorname{supp} f}, \\
& f_{x} \in \mathscr{S}^{\prime}\left(\mathbf{R}^{n}\right), \operatorname{supp} g_{x}=\overline{\operatorname{supp} g},
\end{aligned}
$$




$$
\hat{f}_{x} \hat{g}_{x} \in \mathscr{S}^{\prime}\left(\mathbf{R}^{n}\right), \operatorname{supp}(f * g)_{x}=\overline{\operatorname{supp} f * g} \text {. }
$$

Hence $f_{x}$ and $g_{x}$ have compact support, for these values of $x$. But then the Titchmarsh support theorem in $\mathbf{R}^{n}$ (see for instance Hörmander [5, Theorem 4.3.3]) implies that

$$
\text { ch } \operatorname{supp} f_{x} * g_{x}=\operatorname{ch} \operatorname{supp} f_{x}+\operatorname{ch} \operatorname{supp} g_{x},
$$

and it remains to prove that

$$
f_{x} * g_{x}=(f * g)_{x}
$$

Here $f_{x} * g_{x}$ is, of course, convolution in ordinary distribution sense. Since $f_{x}$ and $g_{x}$ have compact support, $\hat{f}_{x}$ and $\hat{g}_{x}$ are continuous almost everywhere on $\mathbf{R}^{n}$ and

$$
\mathscr{F}\left(f_{x} * g_{x}\right)=\hat{f}_{x} \hat{g}_{x}
$$

The Fourier transform of the right hand member of (9) is by Definition 1

$$
(\hat{f} \hat{g})_{x}=\hat{f}_{x} \hat{g}_{x}
$$

and (9) is proved.

Parseval's relation shows that Definition 1 is applicable in the case when $f, g \in l^{2}\left(\mathbf{R}_{d}^{n}\right)$, and that

$$
f * g(\lambda)=\sum_{\nu \in \mathbf{R}_{d}^{\eta}} f(\lambda-v) g(v), \lambda \in \mathbf{R}_{d}^{n} .
$$

We have then the following more precise theorem.

Theorem 2. Let $f, g \in l^{2}\left(\mathbf{R}_{d}^{n}\right)$, with supp $f$ and $\operatorname{supp} g$ bounded. Then

$$
\operatorname{ch} \operatorname{supp} f * g=\operatorname{ch} \operatorname{supp} f+\operatorname{ch} \operatorname{supp} g \text {. }
$$

Proof. Since Theorem 1 holds, it is enough to discuss the points on the boundary (with respect to the topology of $\mathbf{R}^{n}$ ) of ch supp $f * g$. This is done by induction in $n$. For $n=1$, the theorem is an obvious consequence of (10). So let us assume that $n \geqq 2$ and that the theorem is true for the dimension $n-1$. Let $P$ be any support hyperplane of ch $\operatorname{supp}(f * g)$, and let $P_{1}$ and $P_{2}$ be the corresponding parallel support hyperplanes of ch supp $f$ and ch supp $g$, respectively, such that $P=P_{1}+P_{2}$. (We have here used Theorem 1.) Denote by $f^{\prime}, g^{\prime}$ and $(f * g)^{\prime}$ the functions obtained by multiplying $f, g$ and $f * g$ with the characteristic functions of $P_{1}, P_{2}$ and $P$, respectively. (10) shows that

$$
(f * g)^{\prime}=f^{\prime} * g^{\prime},
$$

and the induction assumption gives easily 
(ch supp $f * g) \cap P=\operatorname{ch} \operatorname{supp}(f * g)^{\prime}=$ ch supp $f^{\prime}+$ ch supp $g^{\prime}=($ ch $\operatorname{supp} f+\operatorname{ch} \operatorname{supp} g) \cap P$.

By varying $P$ we obtain the theorem.

In the case $n=1$, we have the following theorem, which is slightly more general than Theorem 1.

Theorem 3. Let $f=\mathscr{F}^{-1} \hat{f}, g=\mathscr{F}^{-1} \hat{g}$, with $\hat{f}, \hat{g}, \hat{f} \hat{g} \in L^{1}(b \mathbf{R})$, and with supp $f$ and supp $g$ bounded from below. Then

$$
\inf \operatorname{supp} f * g=\inf \operatorname{supp} f+\inf \operatorname{supp} g \text {. }
$$

Proof. It is a known fact (Hoffman [4, pp. 132-133]), that for a function $k$ in $H^{1}(\mathbf{R})$, the exponential function in the product representation of the extension of $k$ to the upper half-plane, determines inf supp $k$, with $k$ defined in accordance with (3). It follows easily from this that if $\hat{\alpha}, \hat{\beta}, \hat{\gamma}=\hat{\alpha} \hat{\beta} \in L^{1}(\mathbf{R})$, then

$$
\inf \operatorname{supp} \gamma=\inf \operatorname{supp} \alpha+\inf \operatorname{supp} \beta \text {, }
$$

if the terms to the right are $>-\infty$. By (2) and Lemma 1, the assumptions of the theorem imply that (11) holds with

$$
\hat{\alpha}(t)=\hat{f}_{x}(t)(i+t)^{-2}, \hat{\beta}(t)=\hat{g}_{x}(t)(i+t)^{-2},
$$

for almost every $x$. Easy considerations show that this implies

$$
\inf \operatorname{supp}(f * g)_{x}=\inf \operatorname{supp} f_{x}+\inf \operatorname{supp} g_{x},
$$

for almost every $x$, and then Lemma 1 gives the desired result.

Remark. In the case $f \in l^{2}\left(\mathbf{R}_{d}\right), g \in l^{2}\left(\mathbf{R}_{d}\right)$ Theorem 1 is a consequence of Helson's theory of cocycles [3]. (See Helson [2, p. 480].)

\section{Generalized Titchmarsh theorems}

Let $\Omega \subseteq \mathbf{R}_{\mathrm{d}}^{n}$, with $\Omega$ open in the topology of $\mathbf{R}^{n}$. If $f$ is a function on $\Omega$ such that, for every $K \subseteq \Omega$ with $K$ compact in $\mathbf{R}^{n}, f$ coincides on $K$ with a function in $\mathscr{F}^{-1} L^{1}$, we say with a slight abuse of language that $f$ is in $\mathscr{F}^{-1} L^{1}$ locally on $\Omega$. If $g^{1}, g^{2} \in \mathscr{F}^{-1} L^{1}$, and if both $g^{1}$ and $g^{2}$ coincide with $f$ on $K$, Lemma 1 applied to $g^{1}-g^{2}$ shows that $g_{x}^{1}$ and $g_{x}^{2}$ coincide on the interior of $K$, for almost every $x$. Hence it is possible to extend the mappings $f \rightarrow f_{x}$, for almost every $x$, in Section 1, to mappings from the family of functions locally in $\mathscr{F}^{-1} L^{1}$ on $\Omega$ to $\mathscr{D}^{\prime}(\Omega)$ in such a way that the relation $g=f$ on an open set $\Omega^{\prime} \subseteq \Omega$, implies that $f_{x}=g_{x}$ on $\Omega^{\prime}$, for almost every $x$. The following lemma is then an obvious extension of Lemma 1 .

Lemma 2. Let $f$ be locally $\mathscr{F}^{-1} L^{1}$ on $\Omega$. Then 


$$
\operatorname{supp} f_{x}=\overline{\operatorname{supp} f}
$$

for almost every $x \in b \mathbf{R}^{n}$.

We will in the following assume that $n=1$. Let $w$ be a decreasing positive function on $\mathbf{R}_{d}$. We define

$$
w_{1}(\lambda)=1 / w(-\lambda), \lambda \in \mathbf{R}_{d}
$$

$l_{w}^{2}\left(\mathbf{R}_{d}\right)$ is the space of all $f$ with $f w \in l^{2}\left(\mathbf{R}_{d}\right)$. For every complex-valued $f$ on $\mathbf{R}_{d}, f^{n}$ denotes the product of $f$ and the characteristic function of $(n, n+1]$.

Let $f \in l_{w}^{2}\left(\mathbf{R}_{d}\right), g \in l_{w_{1}}^{2}\left(\mathbf{R}_{d}\right)$. Then, by Parseval's relation,

$$
\sum_{n \in \mathbf{Z}} \int_{b \mathbf{R}}\left|\widehat{f^{n}}(x)\right|^{2} d x w(n+1)^{2}=\sum_{n \in \mathbb{Z}} \sum_{\lambda \in \mathbb{R}_{d}}\left|f^{n}(\lambda)\right|^{2} w(n+1)^{2}<\infty
$$

and

$$
\sum_{n \in \mathbb{Z}} \int_{b \mathbb{R}}\left|\widehat{g^{n}}(x)\right|^{2} d x w_{1}(n+1)^{2}=\sum_{n \in Z} \sum_{\lambda \in \mathbb{R}_{d}}\left|g^{n}(\lambda)\right|^{2} w_{1}(n+1)^{2}<\infty
$$

(12) and (13) show that (2) holds with $f$ replaced by any of the functions

$$
\sum_{n \in \mathbf{Z}}\left|\widehat{f^{n}}\right|^{2} w(n+1)^{2} \text { and }\left.\sum_{n \in \mathbf{Z}} \widehat{\mid g^{n}}\right|^{2} w_{1}(n+1)^{2}
$$

and we obtain, for almost every $x$,

$$
\begin{aligned}
& \sum_{n \in Z} \int_{\mathbf{R}}\left|\left(\widehat{f^{n}}\right)_{x}(t)\right|^{2}(1+|t|)^{-2} d t w(n+1)^{2}<\infty, \\
& \sum_{n \in \mathbf{Z}} \int_{\mathbf{R}}\left|\left(\widehat{g^{n}}\right)_{x}(t)\right|^{2}(1+|t|)^{-2} d t w_{1}(n+1)^{2}<\infty .
\end{aligned}
$$

The Schwartz inequality shows that $(f * g)(\lambda)$ is well defined by $(10)$, if $\lambda \geqq 0$. Let $N \in \mathbf{Z}, N \geqq 4$. (12) and (13) show that

$$
h=\sum_{n \in Z}\left(f^{n-2} * g^{N-n}+f^{n-1} * g^{N-n}+f^{n} * g^{N-n}\right)
$$

belongs to $\mathscr{F}^{-1} L^{1}$, and

$$
h=f * g, \text { on }(N-1, \mathrm{~N}+1] .
$$

By (14) and (15) we have, for almost every $x$,

$$
h_{x}=\sum_{n \in Z}\left\{\left(f^{n-2}\right)_{x} *\left(g^{N-n}\right)_{x}+\left(f^{n-1}\right)_{x} *\left(g^{N-n}\right)_{x}+\left(f^{n}\right)_{x} *\left(g^{N-n}\right)_{x}\right\},
$$


with convergence in distribution sense. Note that Lemma 1 shows that

$$
\operatorname{supp}\left(f^{m}\right)_{x} \subseteq[m, m+1], \operatorname{supp}\left(g^{m}\right)_{x} \subseteq[m, m+1]
$$

for every $m \in \mathbf{Z}$ and almost every $x$.

Let $\phi \in \mathscr{D}(\mathbf{R})$, with supp $\phi \leqq[0,1 / 3]$. Then an easy calculation, using (16), shows that, for almost every $x$,

$$
h_{x} * \phi * \phi=\left(f_{x} * \phi\right) *\left(g_{x} * \phi\right)
$$

on $(N-1 / 3, N+1)$. The right hand member of this equality is well defined on $[2, \infty)$, since (14) and (15) show that

$$
\int_{\mathbf{R}}\left|f_{x} * \phi(\lambda)\right|^{2} w(\lambda+1)^{2} d \lambda<\infty
$$

and

$$
\int_{\mathbf{R}}\left|g_{x} * \phi(\lambda)\right|^{2} w_{1}(\lambda+1)^{2} d \lambda<\infty
$$

Since $N \geqq 4$ was arbitrary, we have the following conclusion of (17) and Lemma 2 .

Lemma 3. Let $f \in l_{w}^{2}\left(\mathbf{R}_{d}\right), g \in l_{w_{1}}^{2}\left(\mathbf{R}_{d}\right)$, and

$$
f * g(\lambda)=0, \lambda \geqq 0
$$

Then, for almost every $x,(18)$ and (19) hold, and for every $\phi \in \mathscr{D}(\mathbf{R})$, with supp $\phi \subseteq$ $[0,1 / 3]$,

$$
\left(f_{x} * \phi\right) *\left(g_{x} * \phi\right)(\lambda)=0, \text { for } \lambda \geqq 4
$$

We are now in a position to prove the following theorem.

Theorem 4. Suppose that $\log w$ is convex in $(-\infty, 0]$ and concave in $[0, \infty)$, and that

$$
\varliminf_{\lambda \rightarrow-\infty} \frac{\log w(\lambda)}{|\lambda|^{a}}>0, \varlimsup_{\lambda \rightarrow \infty} \frac{\log w(\lambda)}{\lambda^{b}}<0,
$$

where $a>1, b>1,1 / a+1 / b=1$, and where at least one of the limits is infinite. Let $f \in l_{w}^{2}\left(\mathbf{R}_{d}\right), g \in l_{w 1}^{2}\left(\mathbf{R}_{d}\right)$, both not identically vanishing. If

$$
f * g(\lambda)=0, \text { for all } \lambda \leqq 0,
$$

then inf supp $f>-\infty$, inf supp $g>-\infty$.

Proof. By Lemma 3 we obtain, for almost every $x$, that 


$$
\left(f_{x} * \phi\right) *\left(g_{x} * \phi\right)(\lambda)=0
$$

for $\lambda \geqq 4$, if $\phi \in \mathscr{D}(\mathbf{R})$, supp $\phi \leqq[0,1 / 3]$, and that (18) and (19) hold. By Theorem 1 of [1],

$$
\operatorname{supp}\left(f_{x} * \phi\right) \text { and } \operatorname{supp}\left(g_{x} * \phi\right)
$$

are bounded from below unless one of the sets is empty. This implies, by varying $\phi$, that $\inf \operatorname{supp} f_{x}$ and inf $\operatorname{supp} g_{x}$ are finite, for almost every $x$. Hence the same holds, by Lemma 2, for inf supp $f$ and inf supp $g$.

Theorem 5. If inf supp $f>-\infty$, the conclusion of Theorem 4 holds with (20) changed to the weaker condition

$$
\lim _{\lambda \rightarrow \infty} \frac{\log |\log w(\lambda)|-\log \lambda}{\sqrt{\log \lambda}}=\infty
$$

Proof. Here we apply instead Theorem 2 of [1] in the preceding proof.

Let us form the space $l_{w}^{2}\left(\mathbf{R}_{d}^{+}\right)$of all $f$ on $\mathbf{R}_{d}^{+}$with $f w \in l^{2}\left(\mathbf{R}_{d}^{+}\right)$. Both $l_{w}^{2}\left(\mathbf{R}_{d}\right)$ and $l_{w}^{2}\left(\mathbf{R}_{d}^{+}\right)$ are Hilbert spaces. For $a \geqq 0$, (right) translation $T_{a}$ is defined by

$$
T_{a} f(\lambda)=f(\lambda-a)
$$

if $f \in l^{2}\left(\mathbf{R}_{d}\right)$, while

$$
T_{a} f(\lambda)=\left\{\begin{array}{l}
f(\lambda-a), \lambda \geq a, \\
0,0 \leqq \lambda<a,
\end{array}\right.
$$

if $f \in l^{2}\left(\mathbf{R}_{d}^{+}\right) . T_{a}$ is a contraction, if we assume that $w$ decreases. $l_{w}^{2}\left(\mathbf{R}_{d}\right)$ or $l_{w}^{2}\left(\mathbf{R}_{d}^{+}\right)$is called unicellular, if all closed translation-invariant subspaces are of the form

$$
\{f: f(x)=0, \text { if } x \leqq b\} \text { or }\{f: f(x)=0, \text { if } x<b\} .
$$

Theorem 3 is trivially extendable to arbitrary functions which belong locally to $l^{2}$ and have supports bounded from below. By this and Theorems 4 and 5 (cf. the discussion on p. 299 of [1]) we find easily the following.

Theorem 6. $l_{w}^{2}(\mathbf{R})$ and $l_{w}^{2}\left(\mathbf{R}_{d}^{+}\right)$are unicellular, if $w$ satisfies the assumptions of Theorem 4 and Theorem 5 , respectively.

Remark. It is not known whether the results in this paper can be extended to convolutions of functions which are locally in $l^{p}$ and $l^{q}$, where $p \neq 2$, and $p$ and $q$ are conjugate exponents. It would be of particular interest to know whether Theorem 3 holds if the assumption $\hat{f}, \hat{g}, \hat{f} \hat{g} \in l^{1}(b \mathbf{R})$ is changed to 


$$
f \in \mathrm{I}^{1}\left(\mathbf{R}_{d}\right), g \in l^{\infty}\left(\mathbf{R}_{d}\right)
$$

or to the stronger assumption

$$
f \in l^{1}\left(\mathbf{R}_{d}\right), g \in c_{0}\left(\mathbf{R}_{d}\right)
$$

\section{REFERENCES}

1. Y. Domar, Extensions of the Titchmarsh convolution theorem with applications in the theory of invariant subspaces, Proc. London Math. Soc. (3) 46 (1983), 288-300.

2. H. Helson, Cocycles in harmonic analysis, Actes du Congrès international des mathématiciens 1970 (Gauthier-Villars, Paris, 1971).

3. H. Helson, Analyticity on compact abelian groups, Algebras in Analysis (Academic Press, London 1975), 2-62.

4. K. Hoffman, Banach Spaces of Analytic Functions (Prentice-Hall, Englewood Cliffs, N.J., USA 1962).

5. L. Hörmander, The Analysis of Linear Partial Differential Operators. Vol. 1 (Springer, Berlin 1983).

6. E. C. Titchmarsh, The zeros of certain integral functions. Proc. London Math. Soc. (2) 25 (1926), 283-302.

Department of Mathematics

UPPSALA UNIVERSITY

Thunbergsvagen 3, S-752 38

UpPSAla, Sweden 